\title{
CT features associated with EGFR mutations and ALK positivity in patients with multiple primary lung adenocarcinomas
}

\author{
Xiaoyu Han ${ }^{1 \dagger}$, Jun Fan ${ }^{2 \dagger}$, Jin Gu${ }^{1}$, Yumin $\mathrm{Li}^{1}$, Ming Yang ${ }^{1}$, Tong Liu', Nan $\mathrm{Li}^{1}$, Wenjuan Zeng ${ }^{3}$ and Heshui Shi ${ }^{{ }^{*}}$ (D)
}

\begin{abstract}
Background: In multiple primary lung adenocarcinomas (MPLAs), the relationship between imaging and gene mutations remains unclear. This retrospective study aimed to identify the correlation of epidermal growth factor receptor (EGFR) mutations and anaplastic lymphoma kinase (ALK) status with CT characteristics in MPLA patients.
\end{abstract}

Methods: Sixty-seven patients (135 lesions) with MPLAs confirmed by pathology were selected from our institution. All subjects were tested for EGFR mutations and ALK status and underwent chest CT prior to any treatment. The criteria for MPLA definitions closely adhered to the comprehensive histologic assessment (CHA).

Results: Among MPLA patients, EGFR mutations were more common in females $(p=0.002)$, in those who had never smoked $(p=0.010)$, and in those with less lymph node metastasis $(p<0.001)$, and the tumours typically presented with ground-glass opacity $(\mathrm{GGO})(p=0.003)$, especially mixed $\mathrm{GGO}(p<0.001)$, and with air bronchograms $(p=0.012)$. Logistics regression analysis showed that $\mathrm{GGO}(\mathrm{OR}=6.550, p=0.010)$ was correlated with EGFR mutation, while air bronchograms were not correlated with $E G F R$ mutation $(\mathrm{OR}=3.527, p=0.060)$. A receiver operating characteristic (ROC) curve yielded area under the curve (AUC) values of 0.647 and 0.712 for clinical-only or combined CT features, respectively, for prediction of EGFR mutations, and a significant difference was found between them $(p=0.0344)$. ALK-positive status was found most frequently in MPLA patients who were younger $(p=0.002)$ and had never smoked $(p=0.010)$. ALK positivity was associated with solid nodules or masses in MPLAs $(p<0.004)$ on CT scans. Logistics regression analysis showed that solid nodules $(\mathrm{OR}=6.550, p=0.010)$ were an independent factor predicting ALK positivity in MPLAs. For prediction of ALK positivity, the ROC curve yielded AUC values of 0.767 and 0.804 for clinical-only or combined CT features, respectively, but no significant difference was found between them ( $p=0.2267)$.

\footnotetext{
* Correspondence: heshuishi@hust.edu.cn

"This study was designed to assess the association between CT characteristics and EGFR mutations and ALK positivity in the tumours of patients with multiple primary lung adenocarcinomas. This was a retrospective analysis."

${ }^{+}$Xiaoyu Han and Jun Fan contributed equally to this work.

'Department of Radiology, Union Hospital, Tongji Medical College,

Huazhong University of Science and Technology, 1277 Jiefang Rd, Wuhan,

Hubei Province 430022, People's Republic of China

Full list of author information is available at the end of the article
}

(C) The Author(s). 2020 Open Access This article is licensed under a Creative Commons Attribution 4.0 International License, which permits use, sharing, adaptation, distribution and reproduction in any medium or format, as long as you give appropriate credit to the original author(s) and the source, provide a link to the Creative Commons licence, and indicate if changes were made. The images or other third party material in this article are included in the article's Creative Commons licence, unless indicated otherwise in a credit line to the material. If material is not included in the article's Creative Commons licence and your intended use is not permitted by statutory regulation or exceeds the permitted use, you will need to obtain permission directly from the copyright holder. To view a copy of this licence, visit http://creativecommons.org/licenses/by/4.0/ The Creative Commons Public Domain Dedication waiver (http://creativecommons.org/publicdomain/zero/1.0/) applies to the data made available in this article, unless otherwise stated in a credit line to the data. 
(Continued from previous page)

Conclusion: Among MPLA patients, nonsmoking women with less lymph node metastasis and patients with lesions presenting GGO or mixed GGO and air bronchograms on CT were more likely to exhibit EGFR mutations. In nonsmoking patients, young patients with solid lesions on CT are recommended to undergo an ALK status test.

Keywords: Adenocarcinoma, Epidermal growth factor receptor, Anaplastic lymphoma kinase, X-ray computed tomography

\section{Introduction}

Lung cancer has been a leading cause of cancer-related death worldwide for decades. In 1975, Manini and Melamed first introduced the concept and diagnostic criteria for multiple primary lung cancers (MPLCs) $[1,2]$. The proportion of adenocarcinoma in MPLCs is much higher than that of squamous cell carcinoma. With the widespread use of computed tomography (CT) and lung cancer screening, the incidence of MPLAs in patients has been reported as 0.2 to $8 \%$ (3.5 to $14 \%$ in autopsy studies) [3-5]. Although there is no standard for MPLA treatment, the consensus is that different lesions should be managed and staging separately [6, 7]. At present, surgical resection is still the main option for treatment of MPLAs [8, 9]. However, considering a patient's tolerance, it is difficult to resect all MPLA lesions. Randomized clinical trials have demonstrated that in advanced non-small cell lung cancer (NSCLC) progression-free survival (PFS) is longer following treatment with tyrosine kinase inhibitors (TKIs) than following chemotherapy $[10,11]$. Therefore, from the perspective of treatment, genetic testing is still needed for MPLA lesions.

Epidermal growth factor receptor (EGFR) mutations and anaplastic lymphoma kinase (ALK) rearrangement are the two most common druggable targets in lung adenocarcinoma. Recent studies have found that driver mutations (including EGFR and ALK mutations) are highly inconsistent among MPLCs [12, 13], emphasizing the need to separately analyse gene mutation status in multifocal tumours [6]. However, obtaining sufficient tissue from multifocal lung adenocarcinomas for gene mutation analysis before treatment may not be feasible due to inoperability, sampling artefacts or limited biopsy specimen amounts. Therefore, more convenient, noninvasive approaches are needed to augment gene status assessments in patients with nonresectable, multifocal lung adenocarcinomas.

CT is the optimal method to detect and characterize pulmonary tumours. The association between CT features and EGFR mutation or ALK positivity in single primary lung adenocarcinoma (SPLA) has been well established [14-21] and indicates a certain correlation between imaging and gene expression in SPLA. However, to the best of our knowledge, no previous study of MPLAs has evaluated the association of EGFR and ALK mutations with CT features. Therefore, our study aims to explore whether CT characteristics can predict EGFR mutations and ALK positivity in patients with MPLAs.

\section{Materials and methods}

\section{Patients and inclusion criteria}

A total of 1193 patients evaluated by the multidisciplinary thoracic oncology group between January 2014 and February 2019 at the Union Hospital of Tongji Medical College were retrospectively screened. Among them, 107 patients (235 lesions) with at least 2 or more synchronous multiple pulmonary adenocarcinomas according to comprehensive histologic assessment (CHA) [2, 22] during an initial thin-section CT scan were retrospectively evaluated in this study. The inclusion criteria were as follows: (a) pathologically confirmed by surgical resection as lung adenocarcinoma; (b) available pathology reports (including predominant pathological subtype, lymph node metastasis and pleural invasion) with a diagnosis of lung adenocarcinoma; (c) available results for both EGFR mutations and ALK status; and (d) available clinical data, including age, sex, smoking history, and tumour node metastasis (TNM) stage. The exclusion criteria were as follows: (a) thin-section $\mathrm{CT}$ was not available $(n=21)$; (b) the time interval between CT acquisition and surgery was more than 3 months $(n=10)$; (c) preoperative treatment prior to surgery, such as radiation therapy or chemotherapy $(n=6)$; and (d) CT image artefacts that precluded further evaluation $(n=3)$. A total of 67 patients (135 lesions, with 103 synchronous and 22 metachronous lesions) were ultimately included. The medical records of each patient were reviewed retrospectively. Patient clinical characteristics, including age, sex, smoking history, histopathology, nodal involvement, and tumour stage, were recorded. In accordance with $\mathrm{Lv}$ et al. [23], nonsmoking was defined as lifetime exposure to fewer than 100 cigarettes, and the remaining patients were categorized as ever-smokers. TNM staging was based on the IASLC 8th TNM Lung Cancer Staging System [7]. When staging tumours according to the current staging system was difficult, a multidisciplinary team (MDT) was organized to discuss and reach a consensus [24]. This retrospective study was approved by the Institutional Review Board of Union Hospital of Tongji Medical College. All subjects enrolled in this 
study signed a written consent form after being informed of the research details.

Notably, the present study was conducted on a per lesion basis or per patient basis in different situations as follows: clinical characteristics (age, sex, smoking history and lymph node metastasis) were described on a per patient basis. EGFR mutations in an MPLA patient were considered if at least one lesion harboured an EGFR mutation. Similarly, ALK-positive MPLA patients were considered if at least one lesion harboured an ALK-positive lesion. On the other hand, the tumour TNM stage, CT findings, EGFR status, and ALK status were described for each lesion.

\section{MPLA Histopathological analysis and evaluation criteria}

The predominant subtype of lung adenocarcinoma was assessed according to the IASLC/ATS/ERS classification of lung adenocarcinoma. Pathological subtypes of nodules were recorded as the predominant pattern, including atypical adenoma-like hyperplasia (AAH); adenocarcinoma in situ (AIS); microinvasive adenocarcinoma (MIA); invasive adenocarcinoma with predominant lepidic, acinar, papillary, solid, or micropapillary components; and invasive adenocarcinoma variants (including mucinous, foetal, and enteric). Adenocarcinomas with lepidic patterns were defined as minimally invasive adenocarcinomas, adenocarcinomas in situ or invasive adenocarcinomas with lepidic components. Designation of MPLAs in the present study was based on the CHA [2, 22]. MPLAs were histologically indicated as follows: (1) at least one of the multiple lesions was AIS or MIA; (2) the predominant histopathologic pattern was different between multiple lesions; (3) the predominant histopathologic pattern was similar, but there were differences in EGFR or ALK status between multiple lesions; (4) synchronous MPLAs were defined when the tumour-free interval between cancers was $<2$ years, and metachronous lesions were defined when the tumour-free interval between cancers was $\geq 2$ years.

\section{EGFR mutation analysis}

EGFR mutations were analysed with the amplificationrefractory mutation system (ARMS). Primary tumours, lymph nodes, distant metastases, and pleural effusion specimens were excised, aspirated, or biopsied; fixed in $10 \%$ neutral buffered formalin; and then embedded in paraffin. DNA was extracted from the formalin-fixed, paraffin-embedded tissue sections, and a Qiagen FFPE Tissue Kit (Netherlands Roots NV) was used according to the manufacturer's instructions. PCR was carried out with the Mx3000PtM system (Stratagene, La Jolla, USA) using an EGFR 29 Mutations Detection Kit (Amoy Diagnostics, Xiamen, People's Republic of China), and the results were interpreted according to the manufacturer's instructions. Molecular analysis of EGFR mutations was defined as the mutation status of EGFR exons 18, 19,21, 20. Otherwise, other types of EGFR mutations were defined as wild-type EGFR $[4,17]$.

\section{VENTANA ALK immunohistochemistry (IHC) assay}

The VENTANA IHC assay is a fully automated detection method based on the monoclonal antibody D5F3. This assay has been approved by the US FDA and China FDA for ascertaining the eligibility of patients with NSCLC for treatment with ALK TKIs. Formalin-fixed, paraffin-embedded tissue sections with a thickness of $4 \mu \mathrm{m}$ were cut according to the manufacturer's instructions and scored according to the supplied algorithm. The results were dichotomous, where any percentage of positive tumour cells with strong granular cytoplasmic staining indicated ALK positivity, while all other observations were regarded as ALK negativity.

\section{CT image acquisition}

All 67 patients (135 lesions) underwent nonenhanced CT scanning, and 52 patients (105 lesions) also underwent enhanced CT scanning. CT imaging was performed at our institution using a multislice spiral CT system (SOMATOM Definition AS +, Siemens Healthineers, Germany). The scan ranged from the chest inlet to the inferior level of the costophrenic angle. The CT parameters were as follows: detector collimation width, $64 \times 0.6$ $\mathrm{mm}$ and $128 \times 0.6 \mathrm{~mm}$; tube voltage, $120 \mathrm{kV}$. The tube current was regulated by an automatic exposure control system (CARE Dose 4D). Images were reconstructed with a slice thickness of $1.5 \mathrm{~mm}$ and an interval of 1.5 $\mathrm{mm}$. Then, the reconstructed image was transmitted to the workstation and picture archiving and communication systems (PACS) for multiplanar reconstruction (MPR) postprocessing. The mediastinal window (centre, 50; width, 350) and lung window (centre, -600 ; width, 1200) were obtained from the PACS. A nonionic iodine contrast agent (60-80 $\mathrm{mL}$ iohexol $350 \mathrm{mg} / \mathrm{mL}$; Beilu Pharmaceutical Co., Ltd., Beijing, China) was applied to 52 patients via intravenous injection in the elbow, and the dose was $3 \mathrm{~mL} / \mathrm{s}$.

\section{CT image interpretation}

Images were analysed by two radiologists (J.G., thoracic radiologist with 10 years of experience and $\mathrm{X} . \mathrm{H}$, a radiology fellow with 4 years of experience in interpreting CT images). Both radiologists used the Digital Imaging and Communications in Medicine (DICOM) protocol to analyse images from the CT studies without access to clinical and histologic findings but were aware of the presence and sites of the tumours. They assessed the CT features using both axial CT images and MPR images. After they performed separate evaluations, the differences were resolved by consensus. Interpretations of each CT characteristic are presented in Table 1. 
Table $1 \mathrm{CT}$ features for lung adenocarcinoma

\begin{tabular}{|c|c|}
\hline Variable & Definition \\
\hline Type & $\begin{array}{l}\text { Central, tumour located in the segmental or more proximal bronchi; peripheral, } \\
\text { tumour located in the subsegmental bronchi or more distal airway }\end{array}$ \\
\hline Location & $\begin{array}{l}\text { The distribution of each lesion in the lung was recorded, including left upper } \\
\text { lobe, left lower lobe, right upper lobe, right middle lobe and right lower lobe }\end{array}$ \\
\hline $\begin{array}{l}\text { Relationship of location between } \\
\text { multiple lesions in same patient }\end{array}$ & Same lung segment, same lung lobe, ipsilateral lung and heterolateral lung \\
\hline Tumor size & Longest diameter of the tumor in MPR images \\
\hline Ground glass opacity & Ground glass dense nodules with internal vessels and bronchi visible \\
\hline Mix ground glass opacity & Composition of both ground glass opacity and solid \\
\hline Pure ground glass opacity & Composition of ground glass opacity only \\
\hline Texture & $\begin{array}{l}\text { Predominantly solid, Tumour solid component / ground glass component }>0.5 \text {; } \\
\text { Predominantly ground glass opacity, tumour solid diameter / ground glass } \\
\text { diameter } \leq 0.5\end{array}$ \\
\hline Shape & indicated as lobulated, others (round, or oval) \\
\hline Lobulated & The surface of the tumor showed as multiple arc-shaped projections \\
\hline Margin & Evaluated in the lung window, and indicated as smooth, or spiculated \\
\hline Spiculate & $\begin{array}{l}\text { Evaluated in the lung window, and indicated as different degrees of spinous or } \\
\text { burr-like protrusions at the tumour margin }\end{array}$ \\
\hline Margin definition & evaluated in the lung window, and indicated as well-defined, or poor-defined \\
\hline Air bronchogram & Tubelike or branched air structure within the tumour \\
\hline Bubble-like lucency & The $1 \sim 3 \mathrm{~mm}$ of air density area within the mass \\
\hline Margins & Evaluated in the lung window, and indicated as smooth, or spiculated \\
\hline Heterogeneity & $\begin{array}{l}\text { Evaluated in the soft tissue window, and heterogeneity indicated as the } \\
\text { difference of CT values in tumor was greater than } 20 \mathrm{HU}\end{array}$ \\
\hline Pleural attachment & Retraction of the pleura toward the tumour \\
\hline Cavitation & Presence or absence of cavitation \\
\hline Intramodular calcifications & Presence or absence of calcifications \\
\hline Necrosis & Low-density area in the tumour, without enhancement in enhance CT \\
\hline Peripheral emphysema & Presence or absence of peripheral emphysema \\
\hline Peripheral fibrosis & Pulmonary fibrosis around the tumor \\
\hline Vascular convergence & Convergence of vessels to the tumor, applied to the peripheral tumors \\
\hline Enhancement & mild" = $0 \sim 20 \mathrm{HU}$; "moderate" = $20 \sim 40 \mathrm{HU}$, "marked" > $40 \mathrm{HU}$ \\
\hline Lymphadenopathy & $\begin{array}{l}\text { presence or absence of lymphadenopathy thoracic lymph nodes } \\
\text { (hilar or mediastinal) with short-axis diameter greater than } 1 \mathrm{~cm}\end{array}$ \\
\hline
\end{tabular}

\section{Statistical analysis}

Analyses were performed using SPSS Statistics (SPSS, version 21, IBM, Chicago, IL, USA) and MedCalc 16.2.0 (MedCalc Software, Mariakerke, Belgium) software. Clinical characteristics (age, sex, smoking history and lymph node metastasis) are described on a per patient basis. Other clinical and pathological findings, CT features, EGFR status, and ALK status descriptions are described on a per lesion basis. The normality of the distribution was checked using a Kolmogorov-Smirnov test. Normally and nonnormally distributed data and categorical variables are expressed as the mean \pm standard deviation, median (interquartile range) and frequency (percentage), respectively. An independent-sample Student's $t$ test was used to compare two groups of normally distributed variables, and a chi-square test was used to compare categorical variables. Multiple logistic regression analyses were performed to identify independent factors predictive of EGFR or ALK mutation status. The final model was selected by using the enter elimination method, with a cutoff $p$ value of 0.05 . A $p$ value $<0.05$ (two-tailed) was considered to be statistically significant. Receiver operating characteristic curves (ROCs) were constructed for the combined independent factors for predicting EGFR mutations or ALK positivity. Then, a comparison of ROC curves between clinical characteristics alone and clinical characteristics combined with CT signs was performed using the nonparametric approach of DeLong et al. The repeatability test of tumour 
maximum diameter was analysed using the intraclass correlation coefficient (ICC) with a 95\% confidence interval (CI). For other CT signs, interobserver agreement was assessed with the $k$ coefficient [25]. A $p$ value $<0.05$ (twotailed) was considered to be statistically significant.

\section{Results}

\section{Clinical characteristics}

The incidence of MPLAs was 5.6\% (67/1193) in our hospital from January 2014 to February 2019. A total of 67 eligible MPLA patients ( $58 \pm 7$ years, ranging from 35 to 73 years; female/male ratio: $2 / 1$ ) were enrolled, including 135 lesions. In total, $26.9 \%$ of MPLA patients were smokers.

\section{Correlations of EGFR mutations and ALK status with clinical features}

When based on patients $(n=67)$, subjects were divided into an EGFR mutation $(n=43)$ group and a wild-type EGFR group $(n=24)$. As shown in Table 2, EGFR mutations were found more frequently in females $(p=0.011)$, those who had never smoked $(p=0.041)$, and those with less lymph node metastasis $(p<0.001)$, but no significant association was found with age (56 \pm 6 vs $60 \pm 8$ years, $p=0.068)$. When based on lesions $(n=135)$, no differences were found between the EGFR mutation $(n=43)$ group and the wild-type EGFR $(n=24)$ group in terms of TNM stage, pathological subtype or pleural invasion, as shown in Table 3.

On a per patient basis, younger patients $(49 \pm 7$ vs $58 \pm 7$, $p=0.002)$ and those who had never smoked $(p=0.018)$ were more frequently included in the ALK-positive group $(\mathrm{n}=13)$ than in the ALK-negative group $(n=54)$, but no significant association was found with $\operatorname{sex}(p=0.736)$, history of smoking $(p=1.000)$ or lymph node metastasis $(p=0.110)$ (Table 2). On a per lesion basis, the present study cohort was divided into an ALK-positive group ( $n=$ 23) and an ALK-negative group $(n=112)$. ALK-positivity more frequently occurred in tumours with a solid predominant subtype or in mucinous adenocarcinoma $(p<0.001)$. However, no differences were found between the two groups with regard to TNM stage or pleural invasion, as shown in Table 3.

\section{Interobserver agreement in CT interpretation}

The intraclass correlation coefficient for tumour maximum diameter was 0.940 (95\% CI: 0.838, 0.978). Regarding other CT features, the concordance between the two observers was good, with the $k$ coefficients ranging between 0.640 and 0.950 (Table 4).

\section{Correlation of EGFR mutations and ALK rearrangements with CT features}

All CT signs were recorded in terms of prelesions: $10.4 \%$ $(7 / 67)$ of the lesions in the same patient were located in the same lung segment, $15 \%(10 / 67)$ were located in the same lung lobe, $59.7 \%(40 / 67)$ were located in the ipsilateral lung, and $23.9 \%(16 / 67)$ were located in the contralateral lung (Fig. 1).

Table 5 shows the CT feature comparisons of different EGFR and ALK statuses in patients with MPLAs. The present series revealed that ground-glass opacity (GGO) $(p=0.020)$ (Fig. 1) or mixed GGO $(p<0.001)$ (Fig. 2) and air bronchograms $(p=0.012)$ (Fig. 2$)$ were associated with EGFR mutations. No other CT signs were associated with EGFR mutation status (Table 5). Logistics regression analysis showed that $\mathrm{GGO}(\mathrm{OR}=6.550, p=0.010)$ was correlated with EGFR mutations, whereas air bronchograms were not $(\mathrm{OR}=3.527, p=0.060)$. In addition, ALK positivity was associated with solid tumours in MPLAs $(p<0.004)$ (Fig. 3). No other CT signs were associated with ALK rearrangement status (Table 5). Logistics regression analysis showed that solid nodules $(\mathrm{OR}=6.550, p=0.010)$ were an independent factor predicting ALK positivity in MPLAs.

\section{ROC curve analysis}

For prediction of EGFR mutations, receiver operating characteristic curve (ROC) analysis yielded area under the curve (AUC) values of 0.647 and 0.712 for clinical-only or combined CT features, respectively, and a significant difference was found between them $(p=0.344)$ (Fig. 4a).

Table 2 Clinical comparison of multiple primary lung adenocarcinomas in different EGFR and ALK status (in pre-patients)

\begin{tabular}{|c|c|c|c|c|c|c|c|c|}
\hline Variable & $\mathrm{EGFR}+(n=43)$ & EGFR- $(n=24)$ & Total & $P$ & $\mathrm{ALK}+(n=13)$ & ALK- $(n=54)$ & Total & $P$ \\
\hline Age & $56 \pm 6$ & $60 \pm 8$ & $58 \pm 7$ & 0.068 & $49 \pm 7$ & $58 \pm 7$ & $58 \pm 7$ & $0.002^{*}$ \\
\hline Gender & & & & $0.011^{*}$ & & & & 0.099 \\
\hline Male & $10(23)$ & $13(54)$ & 23(34) & & $7(54)$ & $16(30)$ & 23(34) & \\
\hline Female & $33(77)$ & $11(46)$ & $44(66)$ & & $6(46)$ & $35(4)$ & $44(66)$ & \\
\hline History of smoking & $8(19)$ & $10(42)$ & $18(27)$ & $0.041^{*}$ & $2(14)$ & $16(30)$ & $18(27)$ & $0.010^{*}$ \\
\hline Lymph node metastasis & $10(23)$ & $16(67)$ & $26(30)$ & $<0.001^{*}$ & $5(38)$ & $8(15)$ & 26(39) & 0.110 \\
\hline
\end{tabular}

* $P$ values were based on comparisons between the two groups

EGFR epidermal growth factor receptor, $A L K$ anaplastic large-cell lymphoma kinase

EGFR +, EGFR mutation; EGFR-, EGFR wild type mutation; ALK +, ALK positive; ALK-, ALK negative 
Table 3 Pathology comparison of of multiple primary lung adenocarcinomas in different EGFR and ALK status (in pre-lesions)

\begin{tabular}{|c|c|c|c|c|c|c|c|c|}
\hline Variable & $\mathrm{EGFR}+(n=62)$ & EGFR- $(n=73)$ & Total & $P$ & $\mathrm{ALK}+(n=23)$ & $\operatorname{ALK}-(n=112)$ & Total & $P$ \\
\hline TNM stage ${ }^{a}$ & & & & 0.312 & & & & 0.160 \\
\hline$|-| \mid$ & $22(35)$ & $20(27)$ & $42(31)$ & & $10(43)$ & $32(29)$ & $42(31)$ & \\
\hline III-IV & $40(65)$ & $53(73)$ & $93(29)$ & & $13(57)$ & $80(71)$ & $93(68)$ & \\
\hline \multicolumn{9}{|l|}{ Histological subtype } \\
\hline Lepidic predominant ${ }^{b}$ & $15(24)$ & $10(14)$ & 25(19) & 0.118 & $3(13)$ & $22(20)$ & 25(19) & 0.567 \\
\hline other subtype $1^{c}$ & $47(76)$ & 63(86) & $110(81)$ & & $20(87)$ & $90(80)$ & $110(81)$ & \\
\hline Solid or Mucinous & $5(8)$ & $11(15)$ & $16(12)$ & 0.210 & $8(35)$ & $8(7)$ & $16(12)$ & $<0.001^{*}$ \\
\hline other subtype $2^{d}$ & $57(92)$ & $61(84)$ & 118(88) & & $15(65)$ & 103(93) & 118(88) & \\
\hline Acinar & $30(48)$ & $29(40)$ & $59(43)$ & & $7(30)$ & $53(47)$ & $59(44)$ & \\
\hline Papillary & $8(13)$ & $21(29)$ & $29(21)$ & & $5(22)$ & $24(21)$ & $29(21)$ & \\
\hline Solid & $3(5)$ & $8(11)$ & $11(8)$ & & 6(26) & $5(4)$ & $11(8)$ & \\
\hline Mucinous & $1(2)$ & $3(4)$ & $4(3)$ & & 2(9) & $2(2)$ & $4(3)$ & \\
\hline Micropapillary & $1(2)$ & 2(3) & $3(2)$ & & 2(9) & $1(1)$ & $3(2)$ & \\
\hline Sieve & 2(3) & 2(3) & $4(3)$ & & $0(0)$ & 4(3) & $4(3)$ & \\
\hline Pleural invasion & $25(40)$ & $27(37)$ & $52(39)$ & 0.691 & 9(39) & $41(37)$ & $52(39)$ & 0.819 \\
\hline
\end{tabular}

* $P$ values were based on comparisons between the two groups

a TNM staging was based on the IASLC 8th TNM Lung Cancer Staging System

${ }^{b}$ Lepidic predominant includes: adenocarcinoma in situ, minimally invasive adenocarcinoma, and lepidic predominant invasive adenocarcinoma

c Other subtypes 1 include: acinar, papillary, micropapillary, and solid predominant adenocarcinoma, as well as variants of invasive adenocarcinoma

${ }^{\mathrm{d}}$ Other subtypes 2 include: adenocarcinoma in situ, minimally invasive adenocarcinoma, and lepidic, , acinar, papillary, micropapillary, and sieve

predominant adenocarcinoma

EGFR epidermal growth factor receptor, ALK anaplastic large-cell lymphoma kinase, EGFR + EGFR mutation, EGFR- EGFR wild type mutation, ALK + ALK positive,

ALK- ALK negative

Table 4 Analysis of inter-reader agreement percent of concordance and kappa of agreement

\begin{tabular}{llll}
\hline CT features & $N(\%$ of concordance $)$ & Kappa $(95 \% \mathrm{Cl})$ & Kappa interpretation \\
\hline Shape & $125 / 135$ & $0.846(0.742-0.933)$ & Almost perfect \\
Type & $131 / 135$ & $0.950(0.797-0.978)$ & Almost perfect \\
Texture & $127 / 135$ & $0.860(0.749-0.948)$ & Almost perfect \\
Bubblelike lucency & $128 / 135$ & $0.861(0.754-0.956)$ & Almost perfect \\
Margins & $121 / 135$ & $0.759(0.632-0.871)$ & Almost perfect \\
Vascular convergence & $124 / 135$ & $0.813(0.698-0.916)$ & Almost perfect \\
Air bronchogram & $124 / 135$ & $0.820(0.703-0.921)$ & Almost perfect \\
Pleural retraction & $133 / 135$ & $0.969(0.922-1.00)$ & Almost perfect \\
Spiculate & $130 / 135$ & $0.923(0.847-0.984)$ & Almost perfect \\
Calcifications & $128 / 135$ & $0.640(0.335-0.881)$ & Substantial \\
Enhancement degree & $89 / 105$ & $0.634(0.447-0.783)$ & Substantial \\
Lymphadenopathy & $130 / 135$ & $0.881(0.766-0.976)$ & Almost perfect \\
Cavitation & $132 / 135$ & $0.758(0.393-1.00)$ & Almost perfect \\
Heterogeneity & $126 / 135$ & $0.838(0.731-0.924)$ & Almost perfect \\
Peripheral fibrosis & $127 / 135$ & $0.881(0.794 \sim 0.956)$ & Almost perfect \\
Peripheral emphysema & $121 / 135$ & $0.740(0.605 \sim 0.858)$ & Substantial \\
Necrosis & $122 / 135$ & $0.789(0.674-0.893)$ & Almost perfect \\
\hline
\end{tabular}



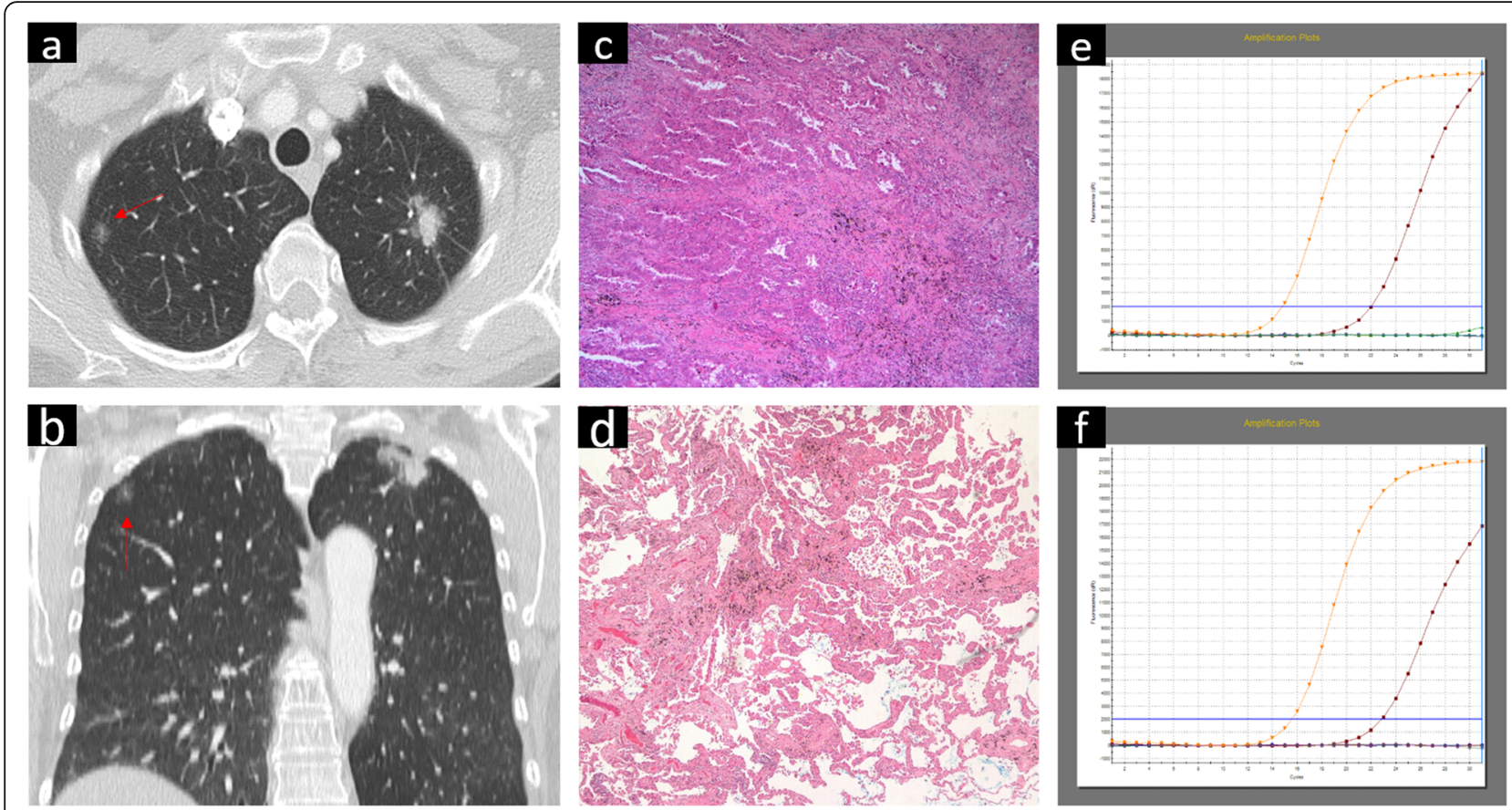

Fig. 1 A 62 year-old female with two concurrent primary lung adenocarcinomas, one was located in the apical posterior segment of the left upper lobe (lesion A) (a), and the other was located in the right upper lobe (lesion B) (b). On CT, lesion A presented as a lobulated, solid nodule with spiculate and pleural traction. Lesion B exhibited light, poorly defined ground glass opacity (GGO). Haematoxylin-eosin (H \& E) staining (c) showed that lesion A was an invasive lung adenocarcinoma with an acinar predominant subtype, and lesion B was a microinvasive lung adenocarcinoma with a lepidic predominant subtype. The ARMS method $(\mathbf{e}, \mathbf{f})$ revealed a 19_del mutation within exon 19 of the EGFR gene in both tumours

For prediction of ALK positivity, the ROC curve yielded AUC values of 0.767 and 0.804 for clinical-only or combined CT features, respectively, but no significant difference was found between them ( $p=0.2267)$ (Fig. 4b).

\section{Discussion}

In this study, we found that the incidence of MPLAs in our hospital from January 2014 to February 2019 was $5.6 \%$, which is consistent with previous reports $(0.2$ to 8\%) [3-5]. The average age of patients with MPLAs in our study was $58 \pm 7$ years old, and the proportion of women was significantly higher than that of men (2:1), similar to a previous study [8]. In terms of patients, the effective EGFR mutation rate in MPLA patients was $64.2 \%$, which was higher than that in single lung adenocarcinoma patients (27-56\%) [26-28]. This result may be because EGFR mutations in an MPLA patient were defined as at least one lesion harbouring an EGFR mutation, which would overestimate the EGFR mutation rate. When based on lesions, the EGFR mutation rate was $45.9 \%$, which was consistent with the results of previous studies [24-26].

When based on patients, EGFR mutations in the MPLA group were more common in women without a smoking history than in the wild-type EGFR group, similar to the clinical characteristics of patients with EGFR mutations in single primary lung adenocarcinoma (SPLA) [26-28]. When based on lesions, no significant difference in the TNM stage of tumours was found between the EGFR mutation group and the wild-type EGFR group, in contrast with the results of $\mathrm{Tu}$ et al. [27]; they found that the EGFR mutation rate in early NSCLC patients was significantly higher than that in advanced NSCLC patients. This difference may be because most of the patients in our study were stage III-IV (68.9\%), while in the Tu et al. series, stage III-IV lung adenocarcinoma only accounted for $22.6 \%$ of the patients. On the other hand, we found that EGFR mutation was less associated with lymph node metastasis, supporting a lower stage in EGFR mutation cases. Previous studies reported that the mutation rate of EGFR in lepidic predominant lung adenocarcinoma was higher than that in adenocarcinoma of other subtypes [16, 26, 29]. In this study, although the EGFR mutation rate in adenocarcinoma with the lepidic predominant subtype (24.2\%) was higher than that in other subtypes (13.7\%), there was no significant difference between them, likely because the incidence of the lepidic predominant subtype was low (18.5\%).

In the present study, most lesions were located in the ipsilateral lung (59.7\%) and in the same lung lobe and the same lung segment, followed by the contralateral 
Table $5 \mathrm{CT}$ features comparison of multiple primary lung adenocarcinomas in different EGFR and ALK status (in pre-lesions)

\begin{tabular}{|c|c|c|c|c|c|c|c|c|}
\hline CT features & EGFR+ $(n=62)$ & EGFR- $(n=73)$ & Total & $P$ & $\mathrm{ALK}+(n=23)$ & $\operatorname{ALK}-(n=112)$ & Total & $P$ \\
\hline Diameter $(\mathrm{cm})^{a}$ & $1.7 \pm 1.3$ & $1.7 \pm 1.6$ & $1.7 \pm 1.5$ & 0.835 & $2.1 \pm 2.1$ & $1.6 \pm 1.3$ & $1.7 \pm 1.5$ & 0.237 \\
\hline Type & & & & 0.118 & & & & 0.305 \\
\hline Central & $15(24)$ & $10(14)$ & $25(19)$ & & $6(26)$ & $19(17)$ & $25(19)$ & \\
\hline Peripheral & $47(76)$ & 63(86) & $110(81)$ & & $17(74)$ & $93(83)$ & 110(81) & \\
\hline Distribution & & & & 0.537 & & & & $0.007^{*}$ \\
\hline left upper lobe & $13(21)$ & $12(16)$ & 25(19) & & $5(22)$ & $20(18)$ & $25(19)$ & \\
\hline left lower lobe & $12(19)$ & $8(11)$ & $20(15)$ & & $1(4)$ & $19(17)$ & $20(15)$ & \\
\hline right upper lobe & $17(27)$ & $21(29)$ & $38(28)$ & & 3(13) & $35(31)$ & $38(28)$ & \\
\hline right middle lobe & $8(13)$ & $12(16)$ & 20(15) & & 2(9) & $18(16)$ & $20(15)$ & \\
\hline right lower lobe & $12(19)$ & $20(27)$ & $32(23)$ & & $12(52)$ & $20(18)$ & $32(23)$ & \\
\hline Texture & & & & $0.002^{*, b}$ & & & & $0.005^{*, b}$ \\
\hline Solid & $35(56)$ & $59(81)$ & $94(70)$ & & $22(96)$ & $72(64)$ & $94(70)$ & \\
\hline GGO & $27(44)$ & $14(19)$ & $41(30)$ & & $1(4)$ & $40(36)$ & $41(30)$ & \\
\hline pGGO & $4(6)$ & $10(14)$ & $14(10)$ & $0.514^{c}$ & $0(0)$ & $14(13)$ & $14(10)$ & $0.070^{c}$ \\
\hline mGGO & $23(37)$ & $4(5)$ & $27(20)$ & $<0.001^{*}$,d,e & $1(4)$ & $26(23)$ & $27(20)$ & $0.021^{* \mathrm{~d}, \mathrm{e}}$ \\
\hline Shape & & & & 0.259 & & & & 0.341 \\
\hline lobulated & $20(32)$ & $31(41)$ & $51(38)$ & & $7(30)$ & $46(41)$ & $51(38)$ & \\
\hline others & $42(68)$ & $42(58)$ & $84(62)$ & & $16(70)$ & $66(59)$ & $84(62)$ & \\
\hline Spiculate & $25(40)$ & $28(37)$ & $53(39)$ & 0.816 & $14(61)$ & $39(35)$ & $53(39)$ & 0.134 \\
\hline Margin definition & & & & 1.000 & & & & 0.453 \\
\hline well-defined & $18(29)$ & $21(29)$ & $39(29)$ & & $5(22)$ & $34(30)$ & $39(29)$ & \\
\hline poor-defined & $44(71)$ & $52(71)$ & $96(71)$ & & $18(78)$ & 78(70) & $96(71)$ & \\
\hline Air bronchogram & $28(45)$ & $18(25)$ & $46(34)$ & $0.012^{*}$ & $5(22)$ & $41(37)$ & $46(34)$ & 0.171 \\
\hline Heterogeneity & $24(39)$ & $35(48)$ & $49(36)$ & 0.358 & $12(52)$ & $47(42)$ & $49(36)$ & 0.369 \\
\hline Pleural attachment & $28(45)$ & $25(34)$ & 53(39) & 0.196 & $8(35)$ & $45(40)$ & $53(39)$ & 0.815 \\
\hline Cavitation & $3(5)$ & 2(3) & $5(4)$ & 1.000 & $3(13)$ & $2(2)$ & $5(4)$ & 1.000 \\
\hline Bubble-like lucency & $17(27)$ & $15(21)$ & $32(24)$ & 0.349 & $5(22)$ & $27(24)$ & $32(24)$ & 1.000 \\
\hline Calcifications & $5(8)$ & $3(4)$ & $8(6)$ & 0.332 & 2(9) & $6(5)$ & $8(6)$ & 1.000 \\
\hline Necrosis & $25(40)$ & $23(32)$ & $48(36)$ & 0.286 & $6(26)$ & $42(38)$ & $48(36)$ & 0.298 \\
\hline Vascular convergence & $15(24)$ & $27(37)$ & $42(31)$ & 0.110 & $6(26)$ & $36(32)$ & $42(31)$ & 0.298 \\
\hline Peripheral fibrosis & $22(35)$ & $21(29)$ & $43(32)$ & 0.404 & $7(30)$ & $36(32)$ & $43(32)$ & 0.873 \\
\hline Peripheral emphysema & $14(23)$ & $20(27)$ & $34(25)$ & 0.521 & $5(22)$ & $2(2)$ & $34(25)$ & 0.676 \\
\hline Enhancement degree & & & & 0.807 & & & & 0.559 \\
\hline mild & $34(69)$ & $41(73)$ & $75(71)$ & & $16(70)$ & $59(70)$ & $75(71)$ & \\
\hline moderate & $12(24)$ & $13(23)$ & $25(24)$ & & $6(26)$ & $19(23)$ & $25(24)$ & \\
\hline marked & $3(7)$ & $2(4)$ & $5(5)$ & & 1(4) & $4(7)$ & $5(5)$ & \\
\hline
\end{tabular}

* $P$ values $<0.05$

a The maximum diameter of the lesion (in centimeters) evaluated on the multiplanar reconstructions (MPRs) with a soft tissue window;

${ }^{\mathrm{b}}$ Comparison between solid and GGO

c Comparison between solid and pGGO

d Comparison between solid and mGGO

e Comparison between pGGO and mGGO

EGFR+ epidermal growth factor receptor mutation, EGFR- EGFR wild-type group, GGO ground-glass opacity, $p G G O$ pure ground-glass opacity, $m G G O$ mix ground-glass opacity

lung (23.8\%). However, Arai et al. [30] demonstrated that $50 \%$ of double primary cancers were located in the contralateral lung, while intrapulmonary metastatic lesions mainly occurred in the same lobes (84.2\%). The studies may differ because all the tumours included in our cases were surgically removed. In terms of tolerance, patients 


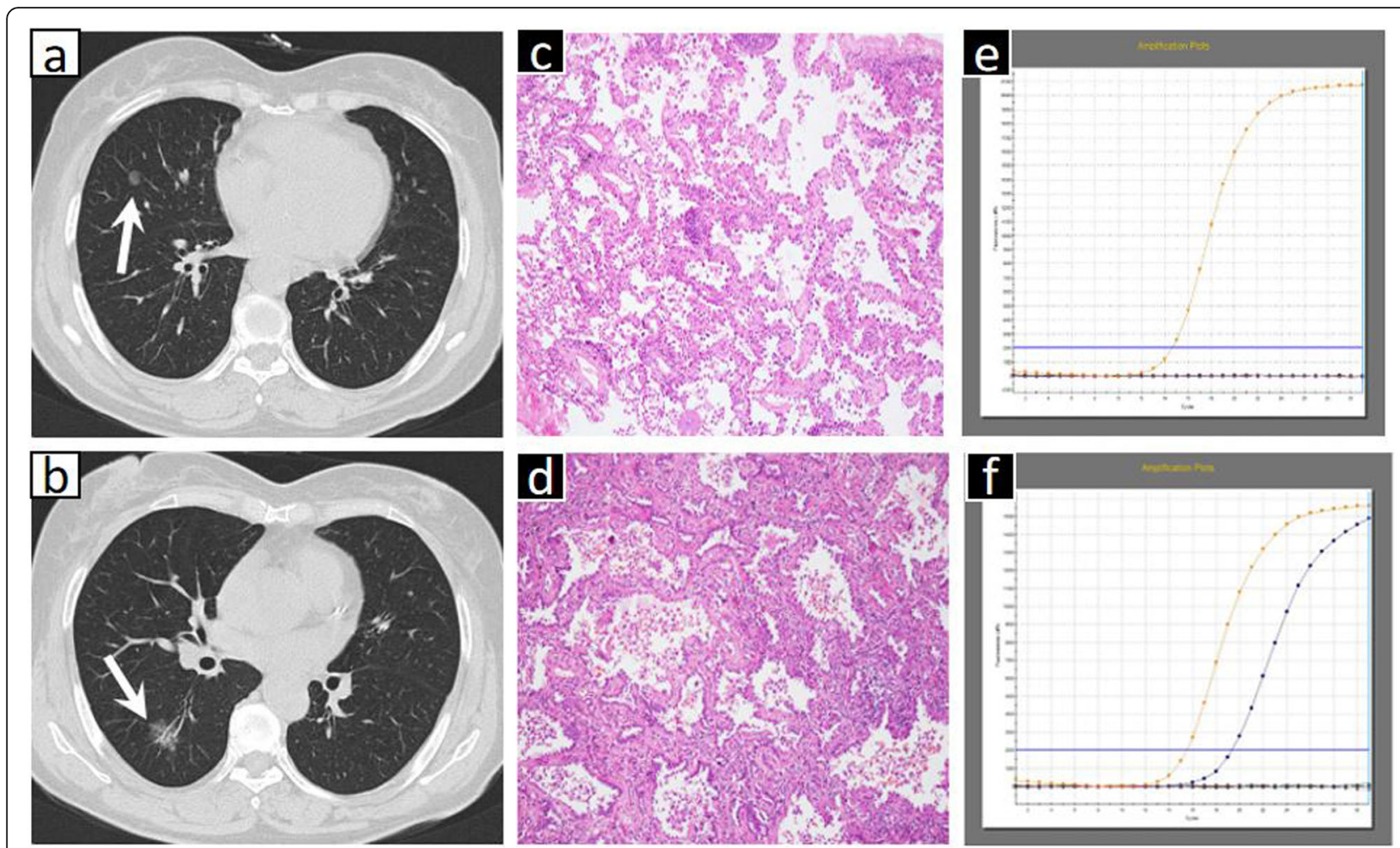

Fig. 2 A 63-year-old female with two concurrent primary lung adenocarcinomas, one in the right middle lobe (lesion A) (a) and one in the right lower lobe (lesion B) (b). By CT, lesion A appeared as a pure ground glass opacity (GGO) nodule, and lesion B exhibited a mixed GGO with a lobulated border. Haematoxylin and eosin (H\&E) staining (c, d) showed different histological adenocarcinoma types, and the ARMS method (e, $\mathbf{f}$ ) revealed a 19_del mutation within exon 19 of the EGFR gene in the lower lobe tumour but not in the middle lobe tumour

exhibited better surgery tolerance when the lesions were located in the ipsilateral lung. We excluded some lesions in the contralateral lung that had not been operated on. Moreover, in the present study, some patients with inoperable lesions are still being followed up.

Previous studies [14, 17, 18, 26, 31] have analysed the potential relationship between the imaging signs of SPLA and EGFR mutation. Among CT features, most of the studies concentrated on the correlation between GGO and EGFR mutation. The present study found that EGFR mutations more frequently showed GGO in CT, consistent with the results of most previous studies in SPLA $[14,15,26]$. This finding may be due to the inverse relationship between EGFR replication and the percentage of GGO on CT [32,33]. In addition, in our study, mixed GGO (mGGO) lesions were more susceptible to EGFR mutations than pure GGO (pGGO) lesions. A possible explanation for the above phenomenon may be that EGFR mutations can promote the conversion of pure GGO to mixed GGO [34]. In addition, this study revealed that EGFR mutations in MPLAs are more common in air bronchograms on CT, which is consistent with previous studies in SPLA [14, 19]. The air bronchograms showed that certain tumours had not yet invaded the bronchus, suggesting a weak aggressiveness of tumours with EGFR mutation. However, no correlation was found between other CT features and EGFR mutations in MPLA lesions, such as tumour size [16], lobulation [14], spicules [15], and pleural attachment [19], as detected in SPLA. These differences occurred not only in our series and previous studies but also differ between previous studies. Possible explanations for the above differences are the different study designs and the demographic features. ROC curve analysis for predicting EGFR mutation showed that the use of clinical combined CT features was significantly superior to use of clinical variables only. Therefore, we may reasonably consider that MPLA tumours with EGFR mutations have imaging patterns similar to those of single lung adenocarcinomas, which emphasizes the need to apply CT features to predict EGFR mutation in MPLA lesions that cannot be biopsied.

ALK positivity has been identified in 0.4 to $13.5 \%$ of unselected NSCLC patients $[35,36]$. In this study, patients with MPLAs had a slightly higher ALK-positive rate $(17.0 \%)$, which may be due to ALK positivity being more frequently found in advanced lung adenocarcinoma [37]. Most of the MPLA patients in our study had high TNM stages (III-IV, 86.6\%), indicating that ALKpositive status was related to advanced tumour stage. 

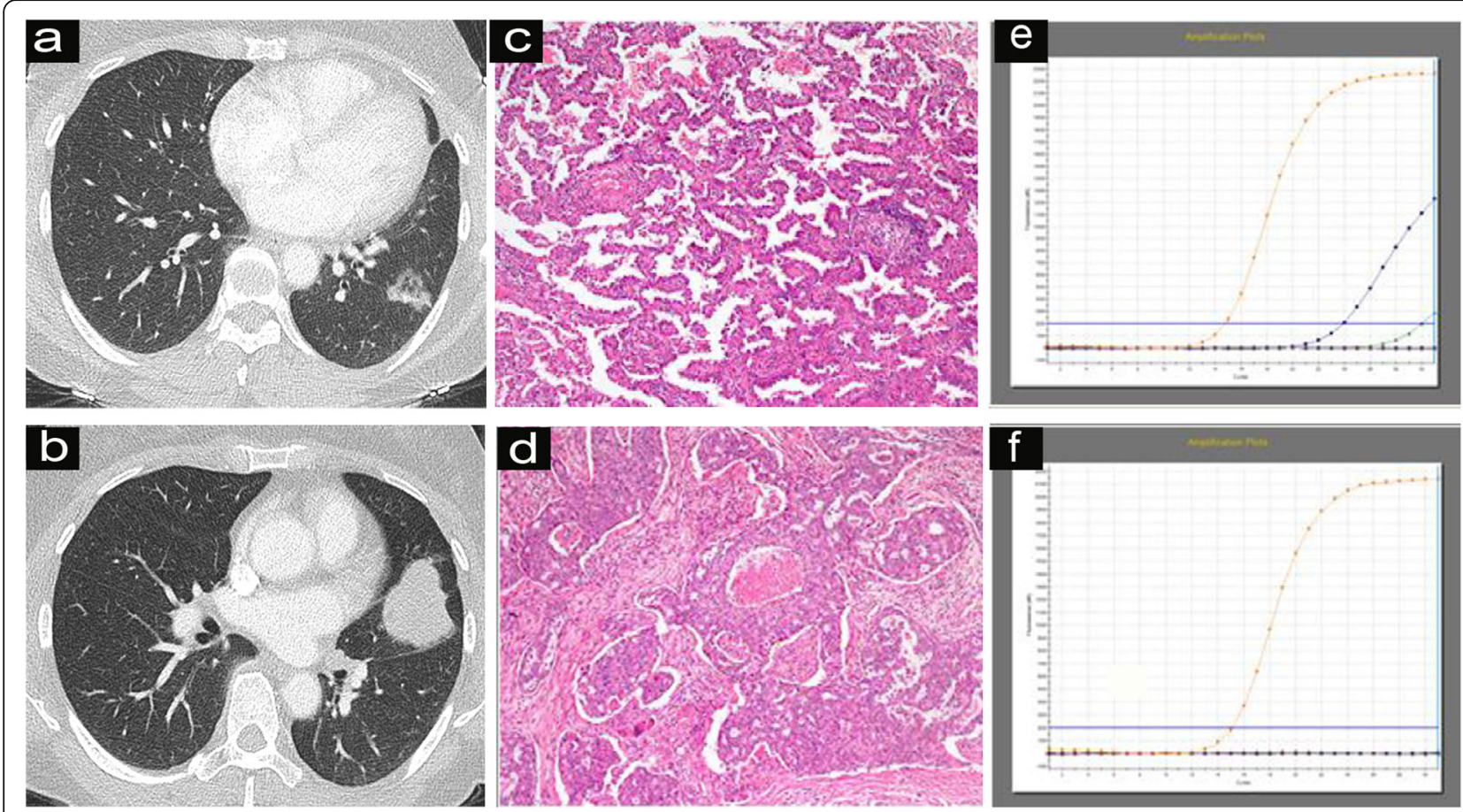

Fig. 3 A 56-year-old female with two concurrent primary lung adenocarcinomas, one in the left lower lobe (lesion A) (a) and one in the left upper lobe (lesion B) (b). Lesion A was a well to moderately differentiated adenocarcinoma and appeared as a mixed GGO nodule on CT. Lesion B was an adenocarcinoma and appeared as a solid mass with a lobulated border on CT. Haematoxylin and eosin (H\&E) staining showed (c) papillary patterns for T1 but (d) solid and cribriform predominant patterns for T2. ARMS-PCR analysis identified an EGFR mutation in (e) T1 but not in (f) T2. IHC and fluorescence in situ hybridization (FISH) showed positive and negative results, respectively, for ALK in T1 but positive results in T2
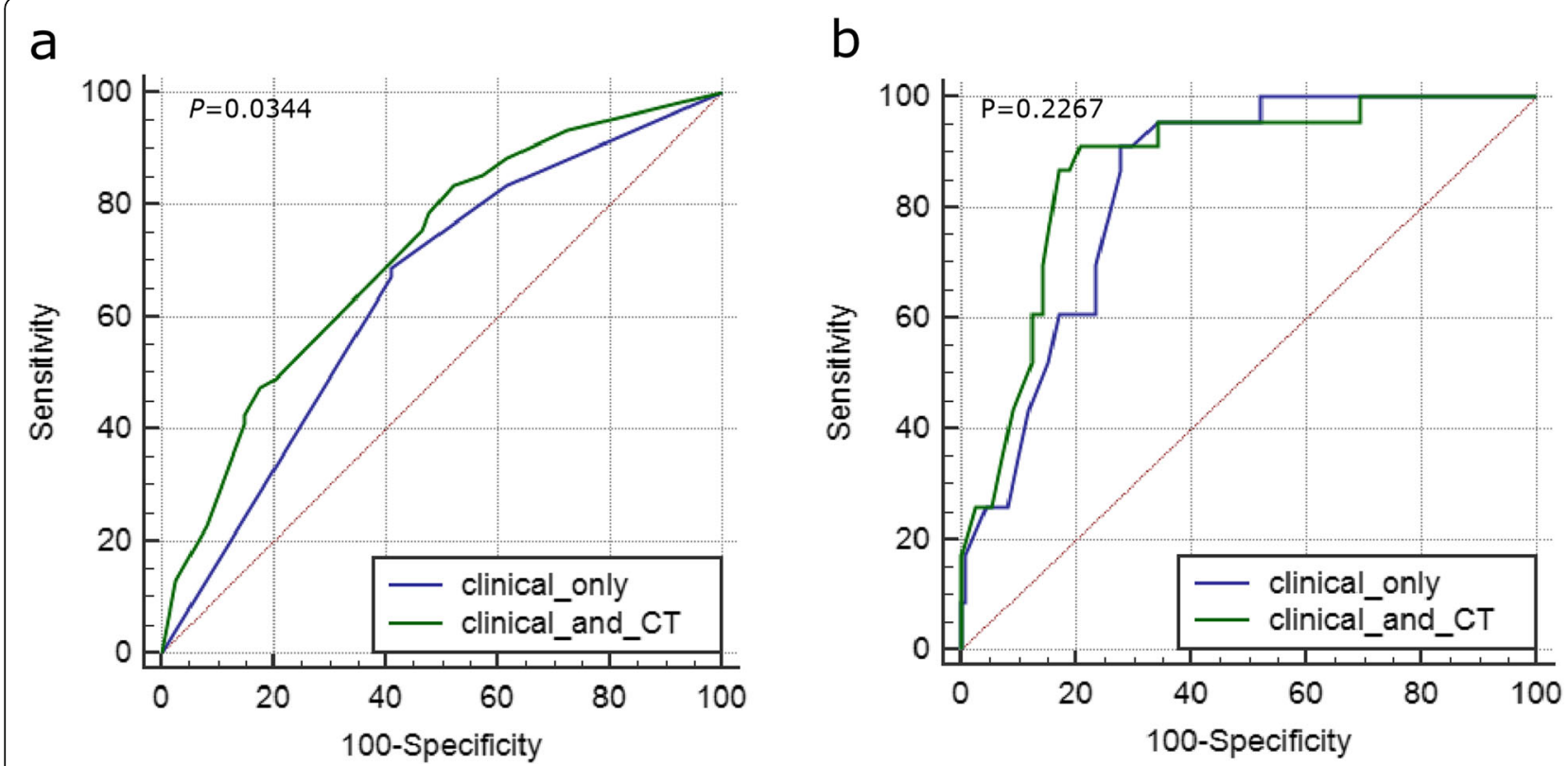

Fig. 4 Receiver operating curve (ROC) curve for EGFR mutation (a) or ALK positivity (b) prediction in MPLAs using clinical features alone or combined with CT signs 
Previous studies have reported that ALK-positive patients tend to be younger and are more often never-smokers than patients with non-ALK rearrangement [38]; this was also confirmed in our study. A recent study by Li et al. [39], conducted with a relatively large sample, demonstrated that ALK positivity was more common in the solid predominant subtype of adenocarcinoma. Akihiko et al. [40] reported that NSCLC with ALK positivity commonly exhibits solid or acinar growth patterns, sieving structures, mucous cells (sign or ring cells) and abundant extracellular mucus. Similarly, our study found that the rate of ALK positivity is higher in the solid predominant subtype or in mucinous adenocarcinoma.

Regarding CT imaging, previous studies [19, 20, 31, 41] have demonstrated that a larger volume, solid mass, extensive lymph node metastasis, pleural invasion, pleural effusion and distant metastasis are associated with ALK positivity, suggesting a highly aggressive feature. In our series, ALK-positive MPLAs were independently associated with the manifestation of solid nodules or masses without a GGO appearance, in line with the above report $[20,31]$. This can be explained by the pathological results in the present study showing that ALK positivity was related to the solid predominant subtype or mucinous adenocarcinoma, because adenocarcinoma with the solid predominant subtype or mucinous adenocarcinoma hardly presented with GGO on CT. Additionally, Aritoshi et al. [42] found that the prognosis was better for double primary lung cancer patients with two GGO nodules than for those with two solid nodules. These findings indicate that MPLA patients who are ALK positive may have a poor prognosis. However, no correlation was found between other CT features and ALK positivity, which may be due to the small sample size in the present study. For prediction of ALK positivity, the ROC curve yielded AUC values of 0.767 and 0.804 for clinical-only or combined CT features, respectively, but no significant difference was found between them. This result may be because few CT features are related to ALK positivity in the present series, which provides a limited diagnostic value for ALK positivity. However, we believe that more promising results will be found in future in studies with a larger sample.

Our study has limitations. 1) The study was performed at a single centre with a small series of patients, especially in terms of the number of ALK-positive cases (13 patients; 23 lesions); Thus, multicentre studies with a large sample size are needed to verify the conclusions of this study and the subgroup analysis. 2) CT features can not only be used to predict the gene status of MPLAs but also to follow up patients with MPLAs, because these patients will develop more lesions that we cannot be biopsied. However, our cases were collected from 2014 to 2019; therefore, the follow-up time was insufficient. Hence, the follow-up chest CT results were not analysed here. Future long-term studies were required to explore the outcome of these patients whether they behaved like true synchronous $\mathrm{T} 1 / \mathrm{T} 2$ or closer to T1NxM1a. 3) Third, the present study analysed only adenocarcinoma and did not include other histologic subtypes. However, this is understandable, as the majority of EGFR mutations and ALK-positivity cases were found in adenocarcinomas, with an extremely low mutation rate in squamous cell carcinoma $(<5 \%)$ [43]. 4) To ensure the reliability of the obtained pathological samples, all cases included in this study were surgically resected. In addition, we excluded some lesions that were only confirmed by biopsy but were not surgically resected, which inevitably affected the comprehensiveness of the study results. 5) The relationship between CT findings and different EGFR mutation types (exon $18,19,20,21$ ) was not discussed in our study due to the small sample, and Lee et al. [16] demonstrated that the GGO volume percentage was significantly higher in tumours with exon 21 mutations than in tumours with other EGFR mutations. However, our results provide directions for further research.

\section{Conclusion}

In conclusion, among patients with MPLAs, nonsmoking women with less lymph node metastasis and patients who present with GGO and air bronchograms on CT are more susceptible to EGFR mutations. In nonsmoking patients, young patients with solid lesions on CT were recommended for the ALK status test. Therefore, our study can reasonably conclude that MPLA tumours with EGFR mutations and ALK positivity have imaging patterns similar to single lung adenocarcinomas. These results can guide clinical treatment of different nodules in patients with MPLAs and aid in development of the best treatment strategy for those patients. However, because this is a small sample, retrospective study, confirmation of these conclusions in larger prospective studies is needed.

\section{Abbreviations \\ MPLCs: Multiple primary lung cancers; MPLAs: Multiple primary lung adenocarcinomas; SPLA: Single primary lung adenocarcinoma; CT: Computed tomography; EGFR: Epidermal growth factor receptor; ALK: Anaplastic lymphoma kinase; NSCLC: Non-small cell lung carcinoma; TKIs: Tyrosine kinase inhibitors; GGO: Ground-glass opacity; pGGO: Pure GGO; mGGO: Mixed GGO; PFS: Progression-free survival; TNM: Tumour node metastasis; CHA: Comprehensive Histologic Assessment; IASLC: International Association for the Study of Lung Cancer; MDT: Multidisciplinary team; MDT: Multidisciplinary teams; ROC: Receiver operating characteristic; AUC: Area under the curve; ARMS: Amplified drug resistance mutation system; IHC: Immunohistochemistry; FFPE: Formalin-fixed paraffin-embedded; FISH: Fluorescence in situ hybridization}

\section{Acknowledgements}

We would like to thank all colleagues for helping us during the current study. We are also very grateful for all the selfless volunteers who participated in the study. 


\section{Authors' contributions}

Conception and design: Xiaoyu Han, Jun Fan, Heshui Shi. Collection and assembly of data: Xiaoyu Han, Jun Fan, Jin Gu, Ming Yang, Tongtong liu, Na li. Data analysis and interpretation: Yumin li, Xiaoyu Han, Jun Fan, Jin Gu, Wenjun Zeng, Ming Yang. Manuscript writing: All authors. Final approval of the manuscript: All authors. Accountable for all aspects of the work: All authors.

\section{Funding}

No funding was provided for this manuscript.

\section{Availability of data and materials}

The datasets used and/or analysed during the current study are available from the corresponding author on reasonable request.

\section{Ethics approval and consent to participate}

This study was approved by the ethics committee of Tongji Medical College of Huazhong University of Science and Technology. All subjects provided written informed consent

\section{Consent for publication}

Not applicable.

\section{Competing interests}

The authors declare no competing non-financial/financial interests.

\section{Author details}

'Department of Radiology, Union Hospital, Tongji Medical College, Huazhong University of Science and Technology, 1277 Jiefang Rd, Wuhan, Hubei Province 430022, People's Republic of China. ${ }^{2}$ Department of Pathology, Union Hospital, Tongji Medical College, Huazhong University of Science and Technology, 1277 Jiefang Rd, Wuhan, Hubei Province 430022, People's Republic of China. ${ }^{3}$ Department of Clinical Laboratory, Union Hospital, Tongji Medical College, Huazhong University of Science and Technology, Wuhan 430022, China.

\section{Received: 9 January 2020 Accepted: 13 July 2020}

\section{Published online: 20 July 2020}

\section{References}

1. Martini N, Melamed MR. Multiple primary lung cancers. J Thorac Cardiovasc Surg. 1975;92(5):606-12.

2. Girard N, Deshpande C, Lau C, Finley D, Rusch V, Pao W, Travis WD. Comprehensive histologic assessment helps to differentiate multiple lung primary nonsmall cell carcinomas from metastases. Am J Surg Pathol. 2009; 33(12):1752-64.

3. Xiaoyan W, Mingsheng W, Maclennan GT, Abdul-Karim FW, Eble JN, Jones TD, Felix O, Rosana E, Cummings OW, Shaobo Z. Evidence for common clonal origin of multifocal lung cancers. J Natl Cancer Inst. 2009:101(8):560-70.

4. Gazdar AF, Minna JD. Multifocal lung cancers--clonality vs field cancerization and does it matter? J Natl Cancer Inst. 2009;101(8):541-3.

5. Ferguson MK, Demeester TR, Deslauriers J, Little AG, Piraux M, Golomb H. Diagnosis and management of synchronous lung cancers. J Thorac Cardiovasc Surg. 1985:89(3):378-85.

6. Xue X, Xue Q, Wang N, Zhang L, Guo L, Li X, Sun J, Wang J. Early clinical diagnosis of synchronous multiple primary lung cancer. Oncol Lett. 2012: 3(1):234-7.

7. Detterbeck FC, Nicholson AG, Franklin WA, Marom EM, Travis WD, Girard N, Arenberg DA, Bolejack V, Donington JS, Mazzone PJ, Tanoue LT, Rusch WW, Crowley J, Asamura H, Rami-Porta R, Staging I, Factors CP, Advisory B. Multiple Pulmonary Sites, I. Participating, The IASLC Lung Cancer Staging Project: Summary of Proposals for Revisions of the Classification of Lung Cancers with Multiple Pulmonary Sites of Involvement in the Forthcoming Eighth Edition of the TNM Classification. J Thorac Oncol. 2016;11(5):639-50.

8. Yu YC, Hsu P-K, Yeh Y-C, Huang C-S, Hsieh C-C, Chou T-Y, Hsu H-S, Wu Y-C, Huang B-S, Hsu W-H. Surgical Results of Synchronous Multiple Primary Lung Cancers: Similar to the Stage-Matched Solitary Primary Lung Cancers? Ann Thoracic Surgery. 2013;96(6):1966-74.

9. Hamaji M, Allen MS, Cassivi SD, Deschamps C, Nichols FC, Wigle DA, Shen KR. Surgical treatment of metachronous second primary lung cancer after complete resection of non-small cell lung cancer. J Thoracic Cardiovascular Surg. 2013;145(3):683-91.

10. Solomon BJ, Mok T, Kim DW, Wu YL, Nakagawa K, Mekhail T, Felip E, Cappuzzo F, Paolini J, Usari T, Iyer S, Reisman A, Wilner KD, Tursi J, Blackhall $F$, Investigators P. First-line crizotinib versus chemotherapy in ALK-positive lung cancer. N Engl J Med. 2014;371(23):2167-77.

11. Mok TS, Wu YL, Thongprasert S, Yang CH, Chu DT, Saijo N, Sunpaweravong P, Han B, Margono B, Ichinose Y. Gefitinib or carboplatin-paclitaxel in pulmonary adenocarcinoma. N Engl J Med. 2009;361(10):947-57.

12. Wu C, Zhao C, Yang Y, He Y, Hou L, Li X, Gao G, Shi J, Ren S, Chu H, Zhou C, Zhang J, Schmid-Bindert G. High discrepancy of driver mutations in patients with NSCLC and synchronous multiple lung ground-glass nodules. J Thorac Oncol. 2015:10(5):778-83.

13. Liu M, He WX, Song N, Yang Y, Zhang P, Jiang GN. Discrepancy of epidermal growth factor receptor mutation in lung adenocarcinoma presenting as multiple ground-glass opacities. Eur J Cardiothorac Surg. 2016:50(5):909-13.

14. Liu Y, Kim J, Qu F, Liu S, Wang H, Balagurunathan Y, Ye Z, Gillies RJ. CT features associated with epidermal growth factor receptor mutation status in patients with lung adenocarcinoma. Radiology. 2016;280(1):151455.

15. Zhou JY, Zheng J, Yu ZF, Xiao WB, Zhao J, Sun K, Wang B, Chen X, Jiang LN, Ding W, Zhou JY. Comparative analysis of clinicoradiologic characteristics of lung adenocarcinomas with ALK rearrangements or EGFR mutations. Eur Radiol. 2015;25(5):1257-66.

16. Hyun-Ju L, Young Tae K, Hyun KC, Binsheng Z, Yongqiang T, Schwartz LH, Thorsten P, Yoon Kyung J, Doo Hyun C. Epidermal growth factor receptor mutation in lung adenocarcinomas: relationship with $C T$ characteristics and histologic subtypes. Radiology. 2013;268(1):254-64.

17. Sugano M, Shimizu K, Nakano T, Kakegawa S, Miyamae Y, Kaira K, Araki T, Kamiyoshihara M, Kawashima O, Takeyoshi I. Correlation between computed tomography findings and epidermal growth factor receptor and KRAS gene mutations in patients with pulmonary adenocarcinoma. Oncol Rep. 2011; 26(5):1205-11.

18. Glynn C, Zakowski MF, Ginsberg MS. Are there imaging characteristics associated with epidermal growth factor receptor and KRAS mutations in patients with adenocarcinoma of the lung with Bronchioloalveolar features? J Thorac Oncol. 2010;5(3):344-8.

19. Rizzo S, Petrella F, Buscarino V, De Maria F, Raimondi S, Barberis M, Fumagalli C, Spitaleri G, Rampinelli C, De Marinis F, Spaggiari L, Bellomi M. CT Radiogenomic characterization of EGFR, K-RAS, and ALK Mutations in Non-Small Cell Lung Cancer. Eur Radiol. 2016;26(1):32-42.

20. Chang-Min C, Young KM, Hye Jeon H, Jung Bok L, Woo Sung $K$. Advanced adenocarcinoma of the lung: comparison of CT characteristics of patients with anaplastic lymphoma kinase gene rearrangement and those with epidermal growth factor receptor mutation. Radiology. 2015;275(1):272-9.

21. Locatelli-Sanchez M, Couraud S, Arpin D, Riou R, Bringuier PP, Souquet PJ. Routine EGFR molecular analysis in non-small-cell lung Cancer patients is feasible: exons 18-21 sequencing results of 753 patients and subsequent clinical outcomes. Lung. 2013;191(5):491-9.

22. Detterbeck FC, Franklin WA, Nicholson AG, Girard N, Arenberg DA, Travis WD. et al. The IASLC Lung Cancer Staging Project: Background Data and Proposed Criteria to Distinguish Separate Primary Lung Cancers from Metastatic Foci in Patients with Two Lung Tumors in the Forthcoming Eighth Edition of the TNM Classification for Lung Cancer. J Thorac Oncol. 2016;11(5):651-65. https://doi.org/10.1016/j.jtho.2016.01.025.

23. Lv Z, Fan J, Xu J, Wu F, Huang Q, Guo M, Liao T, Liu S, Lan X, Liao S, Geng W, Jin Y. Value of (18)F-FDG PET/CT for predicting EGFR mutations and positive ALK expression in patients with non-small cell lung cancer: a retrospective analysis of 849 Chinese patients. Eur J Nucl Med Mol Imaging. 2018:45(5):735-50

24. Vlahos I. Dilemmas in Lung Cancer Staging. Radiol Clin North Am. 2018 ; 56(3):419-35. https://doi.org/10.1016/j.rcl.2018.01.010.

25. Mchugh ML. Interrater reliability: the kappa statistic. Biochemia Medica. 2012;22(3):276-82

26. Khoo C, Rogers TM, Fellowes A, Bell A, Fox S. Molecular methods for somatic mutation testing in lung adenocarcinoma: EGFR and beyond. Translational Lung Cancer Research. 2015:4(2):126.

27. Guillermo Paez J, Janne PA, Lee JC, Tracy S, Greulich H. EGFR Mutations in Lung Cancer Correlation with Clinical Response to Gefitinib Therapy. Science. 2004;304(5676):1497-500. 
28. Maemondo M, Inoue A, Kobayashi K, Sugawara S, Oizumi S, Isobe H, Gemma A, Harada M, Yoshizawa H, Kinoshita I. Gefitinib or chemotherapy for non-small-cell lung cancer with mutated EGFR. N Engl J Medicine. 2010; 362(25):2380-8.

29. Sakuma Y, Matsukuma S, Yoshihara M, Nakamura Y, Nakayama H, Kameda Y, Tsuchiya E, Miyagi Y. Epidermal growth factor receptor gene mutations in atypical adenomatous hyperplasias of the lung. Modern Pathol. 2007;20(9):967.

30. Arai J, Tsuchiya T, Oikawa M, Mochinaga K, Hayashi T, Yoshiura K, Tsukamoto K, Yamasaki N, Matsumoto K, Miyazaki T, Nagayasu T. Clinical and molecular analysis of synchronous double lung cancers. Lung Cancer. 2012;77(2):281-7.

31. Yuan M, Zhang YD, Pu XH, Zhong Y, Li H, Wu JF, Yu TF. Comparison of a radiomic biomarker with volumetric analysis for decoding tumour phenotypes of lung adenocarcinoma with different disease-specific survival. Eur Radiol. 2017:27(11):4857-65.

32. Park EA, Lee HJ, Kim YT, Chang HK, Kang KW, Jeon YK, Jin MG, Chang HL, Chang MP. EGFR gene copy number in adenocarcinoma of the lung by FISH analysis: investigation of significantly related factors on CT, FDG-PET Histopathol. Lung Cancer. 2009;64(2):179-86.

33. Lee Y, Lee HJ, Kim YT, Kang CH, Goo JM, Park CM, Paeng JC, Chung DH, Jeon YK. Imaging characteristics of stage I non-small cell lung cancer on CT and FDG-PET: relationship with epidermal growth factor receptor protein expression status and survival. Korean J Radiol. 2013;14(2):375-83.

34. Hsu K, Chen KC, Yang TY, Yeh YC, Chou TY, Chen HY, Tsai C. Epidermal growth factor receptor mutation status in stage I lung adenocarcinoma with different image patterns. J Thorac Oncol. 2011;6(6):1066-72.

35. Benjamin S, Marileila VG, Ross CD. ALK gene rearrangements: a new therapeutic target in a molecularly defined subset of non-small cell lung cancer. J Thoracic Oncol. 2009;4(12):1450-4.

36. Rikova K, Guo A, Zeng Q, Possemato A, Jian Y, Haack H, Nardone J, Lee K, Reeves C, Yu L. Global survey of Phosphotyrosine signaling identifies oncogenic kinases in lung Cancer. Cell. 2007;131(6):1190-203.

37. Sanja D. Molecular genetic testing for lung adenocarcinomas: a practical approach to clinically relevant mutations and translocations. J Clin Pathol. 2013;66(10):870-4.

38. Shaw AT, Yeap BY, Mino-Kenudson M, Digumarthy SR, Costa DB, Heist RS, Solomon B, Stubbs H, Admane S, McDermott U, Settleman J, Kobayashi S, Mark EJ, Rodig SJ, Chirieac LR, Kwak EL, Lynch TJ, lafrate AJ. Clinical features and outcome of patients with non-small-cell lung Cancer who harbor EML4-ALK. J Clin Oncol. 2009;27(26):4247-53.

39. Li P, Gao Q, Jiang X, Zhan Z, Yan Q, Li Z, Huang C. Comparison of Clinicopathological features and prognosis between ALK rearrangements and EGFR mutations in surgically resected early stage lung adenocarcinoma. J Cancer. 2019;10(1):61-71.

40. Takamochi K, Nagai K, Yoshida J, Suzuki K, Ohde Y, Nishimura M, Sasaki S, Nishiwaki Y. Pathologic NO status in pulmonary adenocarcinoma is predictable by combining serum carcinoembryonic antigen level and computed tomographic findings. J Thoracic Cardiovascular Surgery. 2001;122(2):325-30.

41. Halpenny DF, Riely GJ, Hayes S, Yu H, Zheng J, Moskowitz CS, Ginsberg MS. Are there imaging characteristics associated with lung adenocarcinomas harboring ALK rearrangements? Lung Cancer. 2014;86(2):190-4.

42. Hattori A, Matsunaga T, Takamochi K, Oh S, Suzuki K. Radiological classification of multiple lung cancers and the prognostic impact based on the presence of a ground glass opacity component on thin-section computed tomography. Lung Cancer. 2017;113:7-13.

43. Pao W, Girard N. New driver mutations in non-small-cell lung cancer. Lancet Oncol. 2011;12(2):175-80

\section{Publisher's Note}

Springer Nature remains neutral with regard to jurisdictional claims in published maps and institutional affiliations.

Ready to submit your research? Choose BMC and benefit from:

- fast, convenient online submission

- thorough peer review by experienced researchers in your field

- rapid publication on acceptance

- support for research data, including large and complex data types

- gold Open Access which fosters wider collaboration and increased citations

- maximum visibility for your research: over $100 \mathrm{M}$ website views per year

At BMC, research is always in progress.

Learn more biomedcentral.com/submissions 